\title{
Slow speed resistance exercise training in children with polyarticular juvenile idiopathic arthritis
}

This article was published in the following Dove Press journal:

Open Access Rheumatology: Research and Reviews

\author{
Sangeeta D Sule \\ Kevin R Fontaine ${ }^{2}$ \\ 'Division of Rheumatology, Children's \\ National Health System, Washington, DC \\ 20010, USA; ${ }^{2}$ Department of Public \\ Health, School of Public Health, \\ University of Alabama at Birmingham, \\ Birmingham, $A B$, USA
}

Background: Juvenile idiopathic arthritis (JIA) is an inflammatory autoimmune disease that can cause severe impairment and disability. Exercise is recommended to preserve joint mobility and function. Our objectives were to assess the safety, feasibility, and effects of slow speed resistance exercise in children with polyarticular JIA.

Methods: Patients were recruited from a pediatric rheumatology clinic at an urban hospital and randomized to exercise or control groups. In the intervention group, slow speed resistance exercise with individualized instruction by a certified trainer was performed 1-2 times per week for 12 weeks. The control group performed home-based aerobic exercise 3 days per week for 12 weeks. Pre and post-body composition measurements by dual-energy X-ray absorptiometry; aerobic fitness by peak oxygen uptake during cycle ergometry; isometric muscle strength; and quality of life measures were obtained.

Results: In the exercise group, $9 / 17$ (53\%) completed any exercise training. Of these nine subjects, five (55\%) completed all 12 weeks of the protocol. In the control group, 8/16 (50\%) reported compliance with the recommended aerobic exercise training at least one time per week. Only 2 subjects (12\%) reported exercising more than once per week. There was no significant difference between pre- and post-measurements in any category in the exercise group. There was also significantly elevated body fat in both groups with only $17 \%$ in the control group and $23 \%$ in the exercise group meeting recommended $<30 \%$ total body fat levels.

Conclusions: Children with JIA participated safely in this resistance exercise protocol. The exercise was well-tolerated with no serious adverse events noted. While individual subjects reported improvement in fatigue and improved energy, there was no statistical difference in pre- and post-exercise measures of body composition or quality of life. Identifying ways to improve adherence and encourage exercise in children with JIA is important.

Keywords: exercise, juvenile arthritis, body composition

\section{Introduction}

Juvenile idiopathic arthritis (JIA) is an inflammatory autoimmune disease that can cause severe impairment and disability. The polyarticular subtype, in which children have at least 5 joints involved, affects $30-50 \%$ of children with JIA. ${ }^{1}$ The joint inflammation and effusions lead to synovial hypertrophy, bone loss, and periarticular muscle wasting. Moreover, affected children experience substantial decreases in strength, muscle and bone cross-sectional area, and bone mineral density. ${ }^{2-5}$ A 2-year longitudinal study found that JIA patients have, on average, $35 \mathrm{~g}$ less bone mineral content and $1.2 \mathrm{~kg}$ less lean body mass, and $2.8 \%$ greater body fat than controls individually matched on age, sex, race, and county of residence. ${ }^{6}$ Moreover, up to $50 \%$
Correspondence: Sangeeta D Sule Children's National Health System, I I I Michigan Avenue NW, Room WI-3I4,

Washington, DC 20010 , USA

$\mathrm{Tel}+\mathrm{I} 2024766689$

Email ssule@childrensnational.org 
of adults who suffered from JIA have decreased bone mass. ${ }^{4}$ The reasons for these effects on body composition are not entirely clear but chronic bouts of inflammation, in conjunction with physical inactivity, resulting in muscle atrophy and impaired contractility of muscle fibers. Imaging studies in JIA suggest that muscle loss precedes bone loss. ${ }^{6,7}$ Thus, muscle loss appears to set in motion a cascade of processes that produce skeletal abnormalities in JIA. Besides the disease itself, the anti-inflammatory (ie, prednisone) and immunosuppressive (ie, tumor necrosis factor- $\alpha$ inhibitors) drugs used to quell the disease process can also have detrimental effects on muscle and bone, magnifying the potentially deleterious long-term health consequences. ${ }^{4-8}$

The American College of Rheumatology recommends exercise for patients with arthritis which includes range of motion exercises to preserve joint mobility, along with low resistance and aerobics exercise to maintain muscle mass, bone health, and fitness. ${ }^{9}$ The American Academy of Pediatrics recommendations for strength training include: a 10-15 mins warm-up and cool-down and 2-3 sets of higher repetitions (8-15) two to three times per week for at least 8 weeks. ${ }^{10}$ However, the few studies that have evaluated exercise in JIA have focused on aerobic exercise. ${ }^{11}$ Data on the effects of resistance exercise in children with JIA is largely lacking, but safety and feasibility in a homebased program have been reported. ${ }^{12,13}$ The rationale for the potential value of resistance exercise for JIA comes from studies of adults with rheumatoid arthritis (RA). ${ }^{14,15}$ RA patients randomized to resistance exercise: 1) increased their strength fourfold versus baseline, 2) reduced their pain by $53 \%$, and 3 ) improved 50-foot walk times (all significant versus controls). ${ }^{14}$ Resistance exercise has also been beneficial for children with cystic fibrosis, cerebral palsy, and burn victims. ${ }^{16-18}$ Since resistance exercise has been shown to be well-tolerated and to have beneficial effects, we sought to test the effects of resistance exercise in a cohort of children with polyarticular JIA.

\section{Patients and methods}

\section{Patients}

The study was reviewed and approved by the Johns Hopkins Institutional Review Board. Written informed consent was obtained from patients and/or guardians, and was conducted in accordance with the Declaration of Helsinki. Patients were recruited from a pediatric rheumatology clinic at an urban hospital. Inclusion criteria included ages $\geq 10$ to $\leq 18$ with polyarticular JIA on stable medical therapy and steroid free for 1 month prior to study enrollment. Subjects were excluded if they were pregnant or breast feeding, in an active flare of arthritis, had cardiac or pulmonary disease, or had cognitive impairment. Participants were stratified by gender and randomized to either control or exercise group.

\section{Intervention}

For subjects randomized to the exercise protocol, slow speed resistance exercise with individualized instruction by a certified trainer was performed 1-2 times per week for 12 weeks. This exercise involves performing movements in a slow and controlled fashion without acceleration in the lifting and lowering of the resistance in order to minimize force on the joints and connective tissues. Three exercises, targeting the largest muscles of the body were performed (Leg Press, Chest Press and Compound Row) using machines manufactured by RenEx (Beachwood, $\mathrm{OH}$, USA). Resistance was gradually increased when the individual could perform 5 repetitions before reaching momentary muscular fatigue (ie, until they could not perform another repetition in good form).

For the attention control group, instructions to perform aerobic exercise, such as walking, for 30 mins per day, 3 days a week for 12 weeks were given to subjects and their parents. This group received monthly phone calls to answer questions and assess compliance.

\section{Measures}

Demographic data including age at enrollment, age of JIA diagnosis, gender, and race were collected at study entry. At baseline and 12-weeks, total body lean and fat mass were measured using dual-energy X-ray absorptiometry (GE Lunar Prodigy, Software V. 13). Body weight and height were also measured at each time point. Body mass index (BMI) was defined as per the Centers for Disease Control and Prevention (CDC) guidelines: normal: $<85$ th percentile; overweight, 85-95th percentile; obese $>95$ th percentile. The CDC recommended levels are $<30 \%$ of total body composition body fat and $>60 \%$ lean muscle mass. ${ }^{19-21}$

Aerobic fitness was assessed by peak oxygen uptake (VO2 peak) during cycle ergometry. A Cardinal Health Vmax metabolic system was used to assess oxygen uptake while subjects pedaled on a stationary bicycle. A Biodex unit was used to measure isometric muscle strength using peak isokinetic torques of elbow extension at 60 and 120 degrees/second and knee extension at 60 and 180 degrees/second. Medication data were also collected at baseline and at 12-weeks. Wong-Baker 
FACES Pain Rating Scale, Kids Fatigue Severity Scale, function ability and quality of life (Childhood Health Assessment Questionnaire; C-HAQ) were also measured baseline and at the end of study. ${ }^{22-24}$

\section{Statistical analyses}

We performed two-sided, paired Student's $t$-test for comparison of pre- and post-intervention mean scores. Fisher's exact tests were used to compare data between exercise and control groups in subjects who completed the study. Significance levels were set at ap-value $<0.05$.

\section{Results}

Participant characteristics are shown in Table 1. There was no significant difference between groups in baseline variables, including disease severity. Only $35 \%$ of patients met the recommended lean muscle mass guidelines from the CDC for healthy adolescents. There was also significantly elevated body fat in both groups with only $17 \%$ in the control group and $23 \%$ in the exercise group having $<30 \%$ body fat levels $(p=0.7)$. The Fatigue Severity Score was high in both groups with a mean of 5 in the control group and 5.3 in the exercise group $(p=0.3)$.

In the exercise group, $9 / 17(53 \%)$ completed any exercise training. Of these nine subjects, five (55\%) completed all 12 weeks of the protocol. One subject completed 6 weeks of supervised resistance exercise; two subjects 7 weeks; and one 9 weeks. Fifty-three percent (9/17) completed post-testing.

In the attention control group, 8/16 (50\%) reported compliance with the recommended aerobic exercise training at least one time per week. Only 2 subjects (12\%) reported exercising more than once per week. Eight subjects $(50 \%)$ completed post-study testing.

There was no significant difference between pre- and post-measurements in any category in the exercise group. Figure 1 shows the trends for VO2 max, BMI, fatigue severity scores, and pain scores pre- and post-intervention. In the attention control group, there were no significant differences between pre- and post-measurements in any category. Comparing post measurements between the exercise and control groups, there was no significant difference in BMI, fat or muscle percent, arm or knee flexion and extension, VO2 max, C-HAQ, FACES, or fatigue severity scores.

There were no significant adverse events noted in either group. Eight subjects in the exercise group noted muscle soreness after exercise that lasted 1-2 days and resolved without intervention. No participants in either group noted worsening of arthritis or needed changes in medical therapy.

\section{Discussion}

Children with JIA participated safely in this resistance exercise protocol. The exercise was well-tolerated with

Table I Baseline characteristics by randomization

\begin{tabular}{|c|c|c|c|}
\hline Characteristic & Control, $\mathbf{N}=16$ & Exercise, $N=17$ & p-value \\
\hline Age, mean & I6.I (2.8) & $14(3.3)$ & 0.1 \\
\hline Gender, \% female & 34 & 36 & 0.9 \\
\hline Race, \% AA & 41 & 40 & 0.9 \\
\hline Body mass index, $\mathrm{kg} / \mathrm{m}^{2}$ & $25.7(6.9)$ & $26.5(6.8)$ & 0.8 \\
\hline$\%$ Overweight or obese & 52 & 47 & 0.3 \\
\hline$\%$ meeting guidelines for lean muscle mass ${ }^{a}$ & 35 & 35 & 1.0 \\
\hline$\%$ meeting guidelines for body fat ${ }^{\mathrm{b}}$ & 17 & 23 & 0.7 \\
\hline Time since arthritis diagnosis, years & $2.4(2.1)$ & $2.4(1.8)$ & 0.9 \\
\hline$\%$ on methotrexate & 29 & 60 & 0.1 \\
\hline$\%$ on biologic agent & 35 & 50 & 0.4 \\
\hline Median active joint count & 0 (range $0-1$ ) & 0 (range $0-3$ ) & 0.9 \\
\hline Median restricted joint count & 0 (range 0-4) & 0 (range $0-8$ ) & 0.8 \\
\hline Median Pain (FACES, range $0-5$ ) & 2 (range $0-3$ ) & I (range 0-3) & 0.07 \\
\hline Fatigue (K-FSS, range I-7) & $5.3(0.8)$ & $4.8(1.4)$ & 0.3 \\
\hline Global assessment (C-HAQ, range $0-3)$ & $0.6(0.5)$ & $0.8(0.6)$ & 0.5 \\
\hline Functional ability (C-HAQ, range $0-3$ ) & $0.7(0.6)$ & $0.7(0.5)$ & 0.8 \\
\hline
\end{tabular}

Notes: Values are means (SD) unless otherwise noted. ${ }^{a}$ Centers for Disease Control guidelines: $>60 \%$ of total body composition lean muscle mass. ${ }^{b}$ Centers for Disease Control guidelines: $<30 \%$ of total body composition body fat.

Abbreviations: FACES, Wong-Baker FACES pain scale; K-FSS, kids fatigue severity scale; C-HAQ, childhood health assessment questionnaire. 


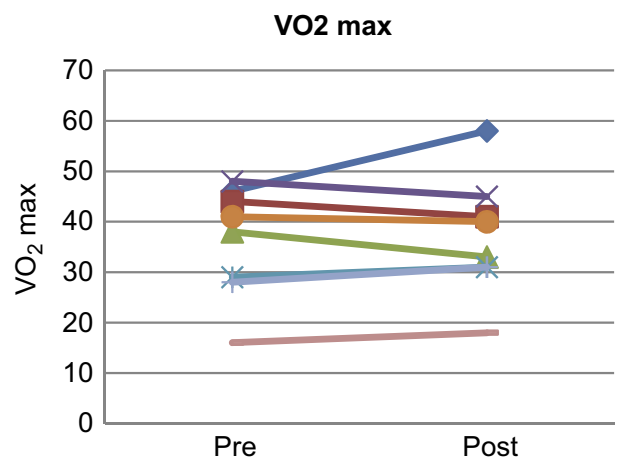

Fatigue severity scale

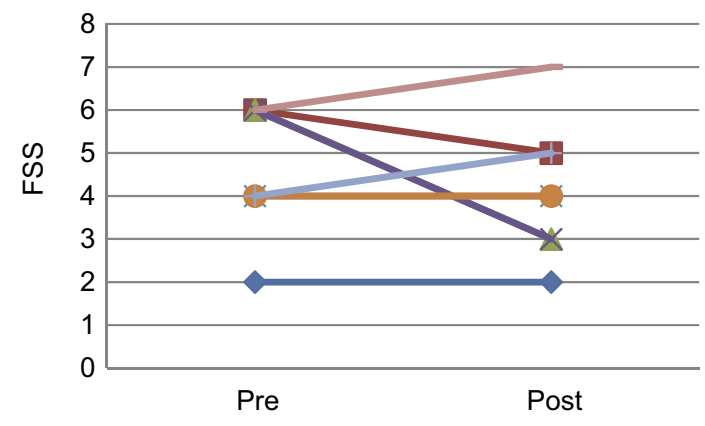

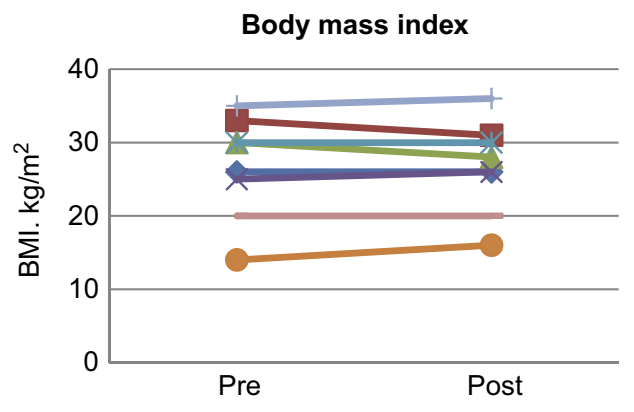

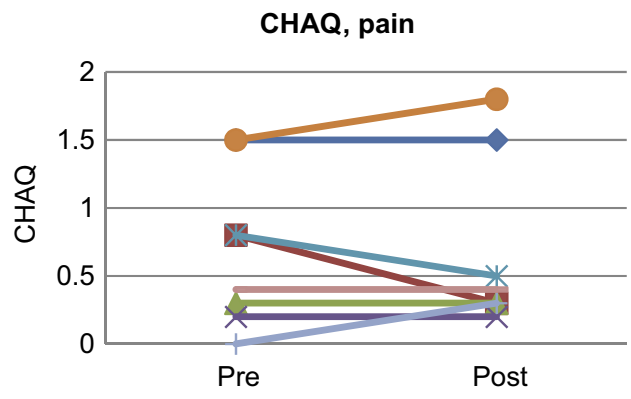

Figure I Effects of resistance exercise intervention $(N=9)$.

no serious adverse events noted. While individual subjects reported improvement in fatigue and improved energy, there was no statistical difference in pre- and postexercise measures of body composition or quality of life.

This study highlights multiple challenges for centerbased, supervised exercise studies in JIA. Both attrition and adherence to the exercise are major challenges in this population. In our study, we had a specialized trainer working with the exercise group who was available on weekends and after-hours. Despite this, only $53 \%$ of subjects participated regularly in the exercise sessions. The most common reason given for attrition from the study was that the exercise program required subjects to come for training in person, rather than participate at home or locations closer to home. However, home-based exercise interventions in this population have also proven to be challenging. In a study by Houghton et al, in a 6-month home-based exercise program of aerobic and resistance exercises, only $47 \%$ of children reported adherence to the program, with adherence decreasing over time. ${ }^{25}$

In our cohort of polyarticular JIA patients, body composition was abnormal. Only $35 \%$ of subjects met the CDC guidelines for recommended lean muscle mass of and $>60 \%$ lean muscle mass. Almost $20 \%$ of our cohort had percent body fat above the recommended CDC guidelines of $<30 \%$ of total body composition body fat. Additionally, almost $50 \%$ of participants in our study were overweight or obese. In a cohort of JIA subjects from Oslo University Hospital, Risum et al reported suboptimal muscle strength and bone mineral density compared to controls, but no differences in body composition with a total body fat of $29.8 \%$ in the JIA polyarticular subjects. These variances in body composition results may be secondary to the different patient populations. ${ }^{26,27}$

Our cohort reported significant fatigue. The overall mean fatigue severity score was 4.8. For comparison, in a cohort of pediatric systemic lupus patients, the mean reported K-FSS score was 3.8. ${ }^{24}$ There was no significant improvement in fatigue scores in either the exercise or control group, but other studies have shown improvement in fatigue with exercise. ${ }^{25}$

Our study has significant limitations. It was difficult to assess the efficacy of resistance exercise because we had a relatively high attrition rate and low adherence. The American Academy of Pediatrics recommendations for strength training include 2-3 sessions per week and our participants met 1-2 times per week which may have not been sufficient for health benefits. One strength of our study is that we had an active control group; however, this group also reported low adherence. We did not assess laboratory 
markers of disease inflammation, such as erythrocyte sedimentation rate or C-reactive protein, before and after the exercise intervention. These results may have helped to determine if exercise had any anti-inflammatory effects. The limitations with regard to adherence suggest that a true test of the effects of resistance exercise remains unknown. Future efforts to identify facilitators and barriers to exercise programs in children with rheumatic diseases are important. Our study shows that exercise is well-tolerated in children with polyarticular JIA and should be encouraged in these patients and rheumatologists should be more uniform in recommending exercise and following up with parents and children.

\section{Acknowledgments}

We sincerely thank the participants and their families who participated in this study. We also thank Michael Bertenthal, dedicated research study coordinator, and Joshua Fontaine, trainer, who both provided invaluable support for the project. We also thank Lori Tucker, MD, for her work as the safety officer for this study. Funding for this project was provided by NIH NIAMS 1R21AR062269-01A1 (Sule).

\section{Disclosure}

The authors have no conflicts of interest to disclose in this work.

\section{References}

1. Prince FHM, Otten MH, van Suijlekom-Smit LWA. Diagnosis and management of juvenile idiopathic arthritis. BMJ. 2010;341.

2. Felin EM, Prahald S, Askew EW, Moyer-Mileur LJ. Musculoskeletal abnormalities of the tibia in juvenile rheumatoid arthritis. Arthritis Rheum. 2007;56:984-994. doi:10.1002/art.22862

3. Roth J, Linge M, Tzaribachev N, Schweiser R, Kuemmerle-Deschuner J. Musculoskeletal abnormalities in juvenile idiopathic arthritis: a 4-year longitudinal study. Rheum (Oxford). 2007;46:1180-1184. doi:10.1093/ rheumatology/kem 100

4. Bechtold S, Roth J. Natural history of growth and body composition in juvenile idiopathic arthritis. Horm Res. 2009;72(suppl 1):13-19. doi: $10.1159 / 000229758$

5. Burnham JM, Shults J, Dubner SE, Sembhi H, Zemel BS, Leonard MB. Bone density, structure, and strength in juvenile idiopathic arthritis. Arth Rheum. 2008;58:2158-2527. doi:10.1002/art.23683

6. Lien G, Selvaag AM, Flatø B, et al. A two-year prospective controlled study of bone mass and bone turnover in children with early juvenile idiopathic arthritis. Arthritis Rheum. 2005;52:833-840. doi:10.1002/ art.21211

7. Roth J, Palm C, Scheunemann I, Ranke MB, Schweizer R, Dannecker GE. Musculoskeletal abnormalities of the forearm in patients with juvenile idiopathic arthritis relate mainly to bone geometry. Arthritis Rheum. 2004;50:1277-1285. doi:10.1002/art.20128

8. Bechtold S, Ripperger P, Dalla Pozza R, Schmidt H, Hafner R, Schwarz HP. Musculoskeletal and functional muscle-bone analysis in children with rheumatic disease using peripheral quantitative computed tomography. Osteoporos Int. 2005;16:757-763. doi:10.1007/ s00198-004-1747-6
9. https://www.rheumatology.org/I-Am-A/Patient-Caregiver/DiseasesConditions/Living-Well-with-Rheumatic-Disease/Exercise-and-Arthritis

10. American academy of pediatrics council on sports medicine and fitness. Pediatrics. 2008;121:835-840. doi:10.1542/peds.20073790

11. Gualano B, Sá Pinto AL, Perondi B, et al. Evidence for prescribing exercise as treatment in pediatric rheumatic diseases. Autoimmuиne Rev. 2010;9:569-573. doi:10.1016/j.autrev.2010.04.001

12. Van Oort C, Tupper SM, Rosenberg AM, Farthing JP, Baxter-Jones AD. Safety and feasibility of a home-based six week resistance training program in juvenile idiopathic arthritis. Pediatr Rheumatol Online J. 2013;11:46-54. doi:10.1186/1546-0096-11-46

13. Myers AM, Beam NW, Fakhoury JD. Resistance training for children and adolescents. Transl Pediatr. 2017;6:137-143. doi:10.21037/ tp.2017.04.01

14. Flint-Wagner HG, Lisse J, Lohman TG, et al. Assessment of a sixteen-week training program on strength, pain, and function in rheumatoid arthritis patients. J Clin Rheumatol. 2009;15:165-171. doi:10.1097/RHU.0b013e318190f95f

15. Hurkmans E, van der Giesen FJ, Vliet Vlieland TP, Schoones J, Van Den Ende EC. Dynamic exercise programs (aerobic capacity and/or muscle strength training) in patients with rheumatoid arthritis. Cochrane Database Syst Rev. 2009;7:CD006853.

16. Selvadurai C, Blimkie C, Meyers N, Mellis C, Cooper J, Asperen V. Randomized controlled study of in-hospital exercise training programs in children with cystic fibrosis. Pediatr Pulm. 2002;33:194-200. doi:10.1002/ppul.10015

17. Morton JF, Brownlee M, McFadyen AK. The effects of progressive resistance training for children with cerebral palsy. Clin Rehabil. 2005;19:283-289. doi:10.1191/0269215505cr804oa

18. Al-Mousawi AM, Williams FN, Micak RP, Jeschke MG, Herndon DN, Suman OE. Effects of exercise training on resting energy expenditure and lean mass during pediatric rehabilitation. J Burn Care Res. 2010;31:400-408. doi:10.1097/BCR.0b013e3181db5317

19. Laurson K, Eisenmann JC, Welk GJ. Development of youth percent body fat standards using receiver operating characteristic curves. $A m$ J Prev Med. 2011;41:S93-S99. doi:10.1016/j.amepre.2011.07.003

20. Laurson KR, Eisenmann JC, Welk GJ. Body fat percentile curves for US children and adolescents. Am J Prev Med. 2011;41:S87-S92. doi:10.1016/j.amepre.2011.06.044

21. Weber DR, Moore RH, Leonard MB, Zemel BS. Fat and lean BMI reference curves in children and adolescents and their utility in identifying excess adiposity compared with BMI and percentage body fat. Am J Clin Nutr. 2013;98:49-56. doi:10.3945/ajen.112.053611

22. Krupp LB, LaRocca NG, Muir J, Stenberg AD. A study of fatigue in systemic lupus erythematosus. $J$ Rheumatol. 1990; 17:1450-1452.

23. Krupp LB, LaRocca NG, Muir-Nash J, Steinberg AD. The fatigue severity scale: application to patients with multiple sclerosis and systemic lupus erythematosus. Arch Neurol. 1989;46:1121-1123.

24. Houghton KM, Tucker LB, Potts J, McKenzie D. Fitness, fatigue, disease activity, and quality of life in pediatric lupus. Arthritis Care Res (Hoboken). 2008;59:537-545. doi:10.1002/art.23534

25. Houghton KM, Macdonald HM, McKay HA, et al. Feasibility and safety of a 6-month exercise program to increase bone and muscle strength in children with juvenile idiopathic arthritis. Pediatr Rheumatol. 2018;16:67. doi:10.1186/s12969-018-0283-4

26. Risum K, Hansen BH, Selvaag AM, Molberg O, Dagfinrud H, Sanner H. Physical activity in patients with oligo-and polyarticular juvenile idiopathic arthritis diagnosed in the era of biologics: a controlled cross-sectional study. Pediatr Rheumatol. 2018;16:64. doi:10.1186/s12969-018-0281-6

27. Risum K, Edvardsen E, Godang K, et al. Physical fitness in patients with oligo- and polyarticular juvenile idiopathic arthritis diagnosed in the era of biologics-a controlled cross-sectional study. Arthritis Care Res. 2018. doi:10.1002/acr.23818 


\section{Publish your work in this journal}

Open Access Rheumatology Research and Reviews is an international, peer-reviewed, open access journal publishing original research, reports, editorials, reviews and commentaries on all aspects of clinical and experimental rheumatology in the clinic and laboratory including the following topics: Pathology, pathophysiology of rheumatological diseases; Investigation, treatment and management of rheumatological diseases; Clinical trials and novel pharmacological approaches for the treatment of rheumatological disorders. The manuscript management system is completely online and includes a very quick and fair peer-review system, which is all easy to use. Visit http://www.dovepress.com/testimonials.php to read real quotes from published authors. 